\title{
Isothermal Titration Calorimetry Resolves Sequential Ligand Exchange and Association Reactions in Treatment of Oleate-capped CdSe Quantum Dots with Alkylphosphonic Acid
}

\author{
Megan Y. Gee, Yi Shen, and Andrew B. Greytak* \\ Department of Chemistry and Biochemistry, University of South Carolina, Columbia, SC 29208 \\ Supporting Information
}

\section{Contents}

1 Thermodynamic models $\quad$ S3

2 Analysis of time-dependent heat signal $\quad$ S5

$\begin{array}{llr}3 & \text { Fitting of heat signals to obtain model parameters } & \text { S8 }\end{array}$

\section{List of Figures}

S1 Effective association constants for ligand exchange . . . . . . . . . . . S6

S2 Distinguishing non-identical sites on the basis of enthalpy . . . . . . . . . . . S6

S3 UV/Vis absorption spectra before and after ligand exchange . . . . . . . . . . . . . . S9

S4 $\quad{ }^{1}$ H NMR of initially purified oleate-capped QDs . . . . . . . . . . . . . . . . . S10

S5 Reference ITC thermograms . . . . . . . . . . . . . . . . . . S11

S6 Detail of ODPA-CdSe $\mid \mathrm{OA}^{-}$ligand exchange with ODPA-to-solvent reference titration . . . S12

S7 Replicate titrations corresponding to Figure $1 \ldots \ldots \ldots \ldots$. . . . . . . . . . . . . . .

S8 Representative basis waveforms . . . . . . . . . . . . . . . . . . S14

S9 $\quad{ }^{1}$ H NMR of oleate-to-phosphonate exchange, vinyl region $\ldots \ldots \ldots \ldots \ldots$. . . . . . S15

S10 Powder X-ray diffraction of CdSe QDs before and after ligand exchange with ODPA . . . . S15

S11 UV/Vis absorption, TEM and PL spectra of Standard Quench (Std) vs. Rapid Quench (RQ) CdSe QDs . . . . . . . . . . . . . . . . . . . . . . . . . . . . . . . . . . . . . .

S12 Effect of quenching rate on ODPA ligand exchange: Representative ITC thermograms . . . . S17

S13 Effect of purification solvent on ODPA ligand exchange: Replicate titrations corresponding to Figure $4 \ldots \ldots \ldots \ldots \ldots \ldots \ldots \ldots \ldots$

S14 Complete waveform fits from time trace analysis . . . . . . . . . . . . . . . S19

S15 Thermodynamic model results for Rapid Quench QDs following THF GPC . . . . . . . . . S20

S16 Thermodynamic model results for Rapid Quench QDs following Toluene GPC . . . . . . S20

*email: greytak@sc.edu 


\section{List of Tables}

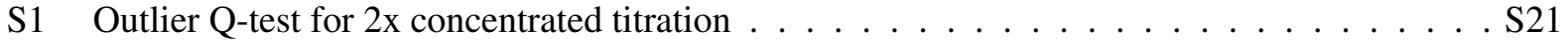

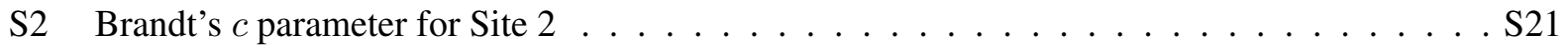




\section{Thermodynamic models}

For a given set of total macromolecule concentration (here, QDs) and total ligand concentration(s), the concentration of bound ligands can be found by solving simultaneous equations for the mass action relationship associated with each binding process, and for the total ligand concentration as a sum of bound and free forms. The heat signal in an ITC experiment then depends on the change in the number of bound species according to the respective binding enthalpies. Solutions are described herein for several simple models of increasing complexity.

\subsection{Ligand association to identical independent sites (Langmuir model)}

The simplest model considers $N$ identical and independent binding sites on each QD. Each site $\mathrm{S}$ is occupied by a ligand $\mathrm{L}$ according to a bimolecular binding equilibrium with equilibrium constant $K$ :

$$
\mathrm{S}+\mathrm{L} \rightleftharpoons \mathrm{SL}
$$

The relevant mass action expression is then

$$
K=\frac{[\mathrm{SL}]}{[\mathrm{S}][\mathrm{L}]}=\frac{\theta}{(1-\theta)[\mathrm{L}]}
$$

where $\theta$ is the average fractional occupation of the sites. By considering a total concentration of macromolecules $[\mathrm{M}]_{\mathrm{t}}$ each with $N$ binding sites, and a total ligand concentration $[\mathrm{L}]_{\mathrm{t}}$ such that

$$
[\mathrm{L}]_{\mathrm{t}}=[\mathrm{L}]_{\mathrm{b}}+[\mathrm{L}]
$$

with $[\mathrm{L}]_{\mathrm{b}}$ and $[\mathrm{L}]$ the concentrations of bound and free ligand, respectively, Equation 2 can be written as

$$
K=\frac{[\mathrm{L}]_{\mathrm{t}}-[\mathrm{L}]}{N[\mathrm{M}]_{\mathrm{t}}-\left([\mathrm{L}]_{\mathrm{t}}-[\mathrm{L}]\right)} \times \frac{1}{[\mathrm{~L}]_{\mathrm{t}}-[\mathrm{L}]_{\mathrm{b}}}
$$

This leads to a quadratic polynominal in [L] that can be solved to obtain the concentration of free ligand, from which all other equilibrium concentrations can be determined:

$$
K[\mathrm{~L}]^{2}+\left(K\left(N[\mathrm{M}]_{\mathrm{t}}-[\mathrm{L}]_{\mathrm{t}}\right)+1\right)[\mathrm{L}]-[\mathrm{L}]_{\mathrm{t}}=0
$$

The ITC thermograms resulting from Langmuir-type association to identical independent size are parameterized by Brandt's $c$ parameter: the shape of the integrated heat response (isotherm) for all experiments with the same value of $c=K N[\mathrm{M}]_{\mathrm{t}}$ is identical and such results can be scaled to overlay one another.

\subsection{Ligand exchange at identical independent sites}

This model considers ligand exchange at identical independent sites that can each be occupied by one of two ligands, as could be appropriate for a simple model of ligand exchange of anionic oleates by phosphonic acids to give free oleic acid and bound, monoanionic phosphonate. The appropriate chemical equation is:

$$
\mathrm{SL}_{1}+\mathrm{L}_{2} \rightleftharpoons \mathrm{SL}_{2}+\mathrm{L}_{1}
$$

with the following mass action expression for the the exchange equilibrium constant $K_{\text {exch }}$ :

$$
K_{\text {exch }}=\frac{\left[\mathrm{SL}_{2}\right]\left[\mathrm{L}_{1}\right]}{\left[\mathrm{SL}_{1}\right]\left[\mathrm{L}_{2}\right]}=\frac{\theta_{2}\left[\mathrm{~L}_{1}\right]}{\theta_{1}\left[\mathrm{~L}_{2}\right]}=\frac{\theta_{2}\left[\mathrm{~L}_{1}\right]}{\left(1-\theta_{2}\right)\left[\mathrm{L}_{2}\right]}
$$


By considering the constraints on the total number of binding sites and total ligand concentrations:

$$
\begin{aligned}
N[\mathrm{M}]_{\mathrm{t}} & =\left[\mathrm{L}_{1}\right]_{\mathrm{b}}+\left[\mathrm{L}_{2}\right]_{\mathrm{b}} \\
{\left[\mathrm{L}_{1}\right]_{\mathrm{t}} } & =\left[\mathrm{L}_{1}\right]_{\mathrm{b}}+\left[\mathrm{L}_{1}\right] \\
{\left[\mathrm{L}_{2}\right]_{\mathrm{t}} } & =\left[\mathrm{L}_{2}\right]_{\mathrm{b}}+\left[\mathrm{L}_{2}\right]
\end{aligned}
$$

and considering the case in which the sites are all initially populated by $\mathrm{L}_{1}$ (in other words $\left[\mathrm{L}_{1}\right]_{\mathrm{t}}=N[\mathrm{M}]_{\mathrm{t}}$ ), it is possible to isolate the concentration of free new ligand $\left[\mathrm{L}_{2}\right]$ as the root of the following quadratic polynomial, from which all other equilibrium concentrations can be determined:

$$
(-K+1)\left[\mathrm{L}_{2}\right]^{2}+\left(-K N[\mathrm{M}]_{\mathrm{t}}+(K-2)\left[\mathrm{L}_{2}\right]_{\mathrm{t}}\right)\left[\mathrm{L}_{2}\right]+\left[\mathrm{L}_{2}\right]_{\mathrm{t}}^{2}=0
$$

However, for large values of the exchange equilibrium constant $K_{\text {exch }}$, the fractional occupation of sites by the new ligand $\left(\theta_{2}\right)$ as a function of $\left[\mathrm{L}_{2}\right]_{\mathrm{t}}$ is similar to that predicted by the Langmuir model for association of $\mathrm{L}_{2}$ to $N$ vacant sites per macromolecule with association constant $K_{\text {eff }}=K_{\text {exch }} /\left(N \times[\mathrm{M}]_{\mathrm{t}}\right)$. This can be seen because close to the saturation point, where $\left[\mathrm{L}_{2}\right]_{\mathrm{t}} \approx N[\mathrm{M}]_{\mathrm{t}}$, much of the initial ligand $\mathrm{L}_{1}$ has been displaced so that $\left[\mathrm{L}_{1}\right] \approx N[\mathrm{M}]_{\mathrm{t}}$. Equation 7 then becomes:

$$
K_{\mathrm{exch}} \approx \frac{\theta_{2} N[\mathrm{M}]_{\mathrm{t}}}{\left(1-\theta_{2}\right)\left[\mathrm{L}_{2}\right]}
$$

Dividing both sides by $N[\mathrm{M}]_{\mathrm{t}}$ gives an expression similar to Equation 2 for an effective association constant $K_{\text {eff }}$ :

$$
K_{\mathrm{eff}}=\frac{K_{\mathrm{exch}}}{N[\mathrm{M}]_{\mathrm{t}}} \approx \frac{\theta_{2}}{\left(1-\theta_{2}\right)\left[\mathrm{L}_{2}\right]}
$$

Figure S1 illustrates the similarity between thermograms for ligand exchange and ligand association with $K_{\text {eff }}$ for large values of the exchange equilibrium constant $K_{\text {exch }}$. In fact, the ITC result approaches that of association to independent sites with Brandt's $c$ parameter simply given by $K_{\text {exch }}$.

\subsection{Ligand association to two types of independent sites}

We now consider the case where each macromolecule has, on average, $N_{1}$ sites with association constant $K_{1}$ and $N_{2}$ sites with association constant $K_{2}$ for the same ligand L, all of which operate independently. In this case, the following relationships must be simultaneously satisfied at equilibrium:

$$
\begin{aligned}
K_{1} & =\frac{\theta_{1}}{1-\theta_{1}} \times \frac{1}{[\mathrm{~L}]} \\
K_{2} & =\frac{\theta_{2}}{1-\theta_{2}} \times \frac{1}{[\mathrm{~L}]} \\
{[\mathrm{L}]_{\mathrm{t}} } & =[\mathrm{L}]_{\mathrm{b} 1}+[\mathrm{L}]_{\mathrm{b} 2}+[\mathrm{L}]=\theta_{1} N_{1}[\mathrm{M}]_{\mathrm{t}}+\theta_{2} N_{2}[\mathrm{M}]_{\mathrm{t}}+[\mathrm{L}]
\end{aligned}
$$

By making appropriate substitutions, it is possible to obtain a cubic polynomial in the free ligand concentration [L] that provides an analytical solution:

$$
\begin{aligned}
K_{1} K_{2}[\mathrm{~L}]^{3}+ & \left(K_{1} K_{2}[\mathrm{M}]_{\mathrm{t}}\left(N_{1}+N_{2}-[\mathrm{L}]_{\mathrm{t}}\right)+K_{1}+K_{2}\right)[\mathrm{L}]^{2} \\
& +\left(\left(N_{1} K_{1}+N_{2} K_{2}\right)[\mathrm{M}]_{\mathrm{t}}-\left(K_{1}+K_{2}\right)[\mathrm{L}]_{t}+1\right)[\mathrm{L}]-[\mathrm{L}]_{\mathrm{t}}=0
\end{aligned}
$$

In the event the association constant $K_{1}$ is much greater than $K_{2}$, the sites of type 1 will tend to fill up first as the total ligand concentration $[\mathrm{L}]_{\mathrm{t}}$ is increased. As these sites become saturated (as $\theta_{1}$ approaches 
1), additional binding will shift to sites of type 2 . In this case, binding to sites of type 1 and 2 may be distinguishable in the ITC heat signal if they have different binding enthalpies $\Delta H_{1}$ and $\Delta H_{2}$.

We note that the ability of isothermal titration calorimetry to distinguish between different sets of binding sites on the basis of their enthalpy offers significant advantages compared to methods that rely primarily on the concentration of free ligand (such as NMR) or that depend on the logarithm of free ligand concentration (such as electrochemical potential measurements) to detect non-identical binding sites. This is illustrated in Figure $\mathrm{S} 2$ for the case of binding with $K_{1}$ and $K_{2}$ differing by factors of $10^{2}$ and $10^{3}$.

\subsection{Ligand exchange and association}

The specific case of ligand exchange at one set of $N_{1}$ sites per macromolecule (replacing $\mathrm{L}_{1}$ with $\mathrm{L}_{2}$, described here by $K_{\text {exch }}$ ) accompanied by simultaneous binding of the new ligand $\mathrm{L}_{2}$ to a separate set of $N_{2}$ independent sites with association constant $K_{2}$, yields the following set of relationships when considered explicitly:

$$
\begin{aligned}
K_{\text {exch }} & =\frac{\theta_{\text {exch }}\left[\mathrm{L}_{1}\right]}{\left(1-\theta_{\text {exch }}\right)\left[\mathrm{L}_{2}\right]} \\
K_{2} & =\frac{\theta_{2}}{\left(1-\theta_{2}\right)\left[\mathrm{L}_{2}\right]} \\
N_{1}[\mathrm{M}]_{\mathrm{t}} & =\left[\mathrm{L}_{1}\right]_{\mathrm{b} 1}+\left[\mathrm{L}_{2}\right]_{\mathrm{b} 1} \\
{\left[\mathrm{~L}_{1}\right]_{\mathrm{t}} } & =\left[\mathrm{L}_{1}\right]_{\mathrm{b} 1}+\left[\mathrm{L}_{1}\right]=\left(1-\theta_{\text {exch }}\right) N_{1}[\mathrm{M}]_{\mathrm{t}}+\left[\mathrm{L}_{1}\right] \\
{\left[\mathrm{L}_{2}\right]_{\mathrm{t}} } & =\left[\mathrm{L}_{2}\right]_{\mathrm{b} 1}+\left[\mathrm{L}_{2}\right]_{\mathrm{b} 2}+\left[\mathrm{L}_{2}\right]=\theta_{\text {exch }} N_{1}[\mathrm{M}]_{\mathrm{t}}+\theta_{2} N_{2}[\mathrm{M}]_{\mathrm{t}}+\left[L_{2}\right]
\end{aligned}
$$

In the above expressions, $\left[\mathrm{L}_{2}\right]_{\mathrm{b} 1}$ and $\left[\mathrm{L}_{2}\right]_{\mathrm{b} 2}$ refer to the concentration of $\mathrm{L}_{2}$ bound to sites of type 1 and 2 respectively, and $\theta_{\text {exch }}$ refers to the fractional occupancy of the ligand exchange site (Site 1) by $\mathrm{L}_{2}$, with exchange equilibrium constant $K_{\text {exch. }}$. Possible binding of $\mathrm{L}_{1}$ to sites of type 2 is neglected.

Though it is possible to simultaneously solve these relationships to obtain $\left[\mathrm{L}_{2}\right]$ at equilibrium, the analytical result is cumbersome, and in the case of this study offers no immediate physical relevance. Given the apparently large value of $K_{\text {exch }}$ for the exchange of oleate by alkylphosphonic acid in our analysis, we have approximated the heat response as arising from the simultaneous binding to two sets (or types) of independent sites (as described in the preceding Section 1.3) with the larger association constant $K_{1}$ being the effective association constant $K_{\text {eff }}$ for the ligand exchange reaction as discussed in Section 1.2, and the smaller association constant $K_{2}$ describing an independent binding interaction of alkylphosphonic acid to a different set of identical sites (Site 2).

\section{Analysis of time-dependent heat signal}

The premise of this analysis is that the power verus time signal following every individual injection can be expressed as a linear combination of a small number of characteristic components associated with the thermodynamic processes that occur when the total concentration of the titrant is incremented. In particular, each process (such as a binding event) has a characteristic time response associated with it based on the rate of reaction convolved with the instrument response function, and the responses to the different injections vary only in the amplitude of each of these components. The amplitudes depend on the extent of reaction for each process in each titration step. By decomposing each observed response into a sum of several characteristic responses, it becomes possible to separate the heat associated with distinct chemical processes over the course of the titration. 

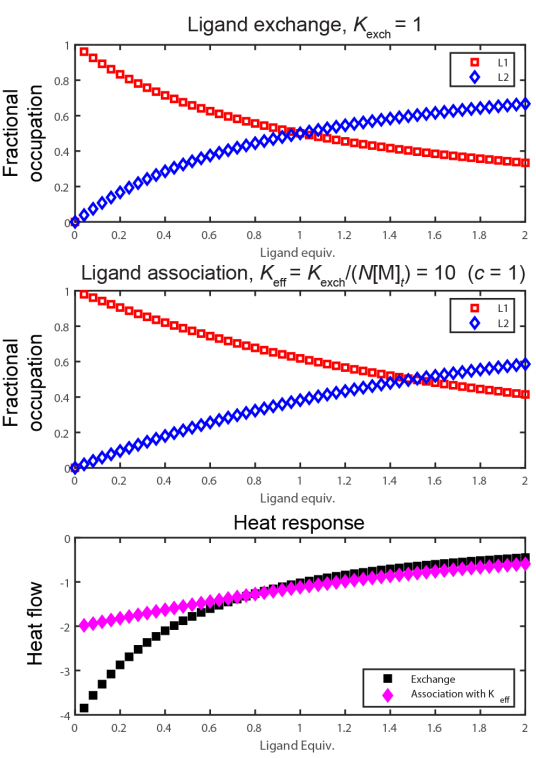

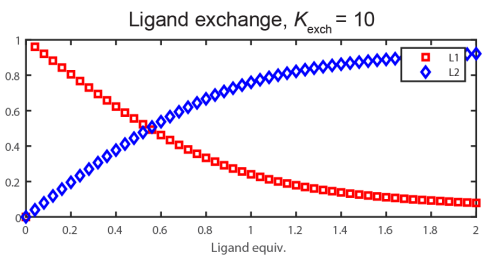

Ligand association, $K_{c \mid l}=K \quad I\left(N[M]_{f}\right)=100(c=10)$

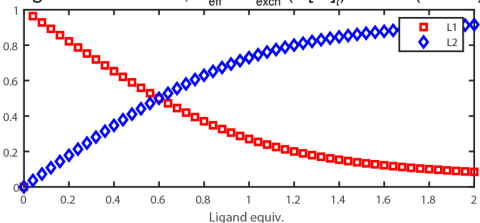

Heat response

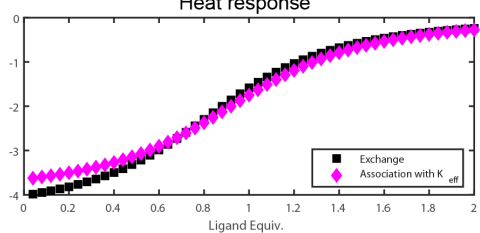

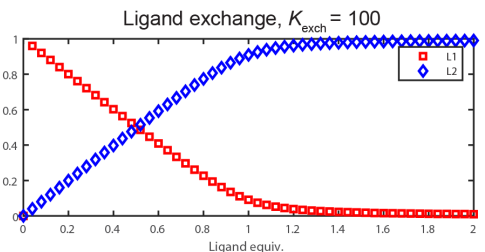

Ligand association, $K_{\text {eff }}=K_{\text {exch }} /\left(N[\mathrm{M}]_{t}\right)=1000(c=100)$

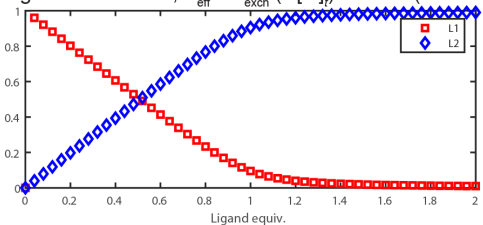

Heat response

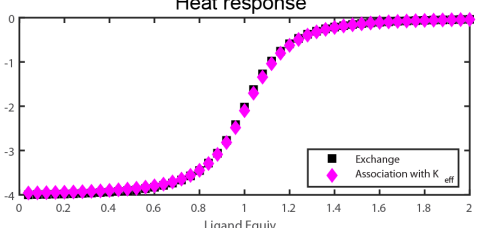

Figure S1: An illustrative model showing how, for large values of the exchange equilibrium constant $K_{\text {exch }}$, a 1:1 ligand exchange at identical independent sites gives results that are quantitatively similar to ligand association with an effective association constant $K_{\text {eff }}$, in the case that the initial concentration of both free ligands is negligible.
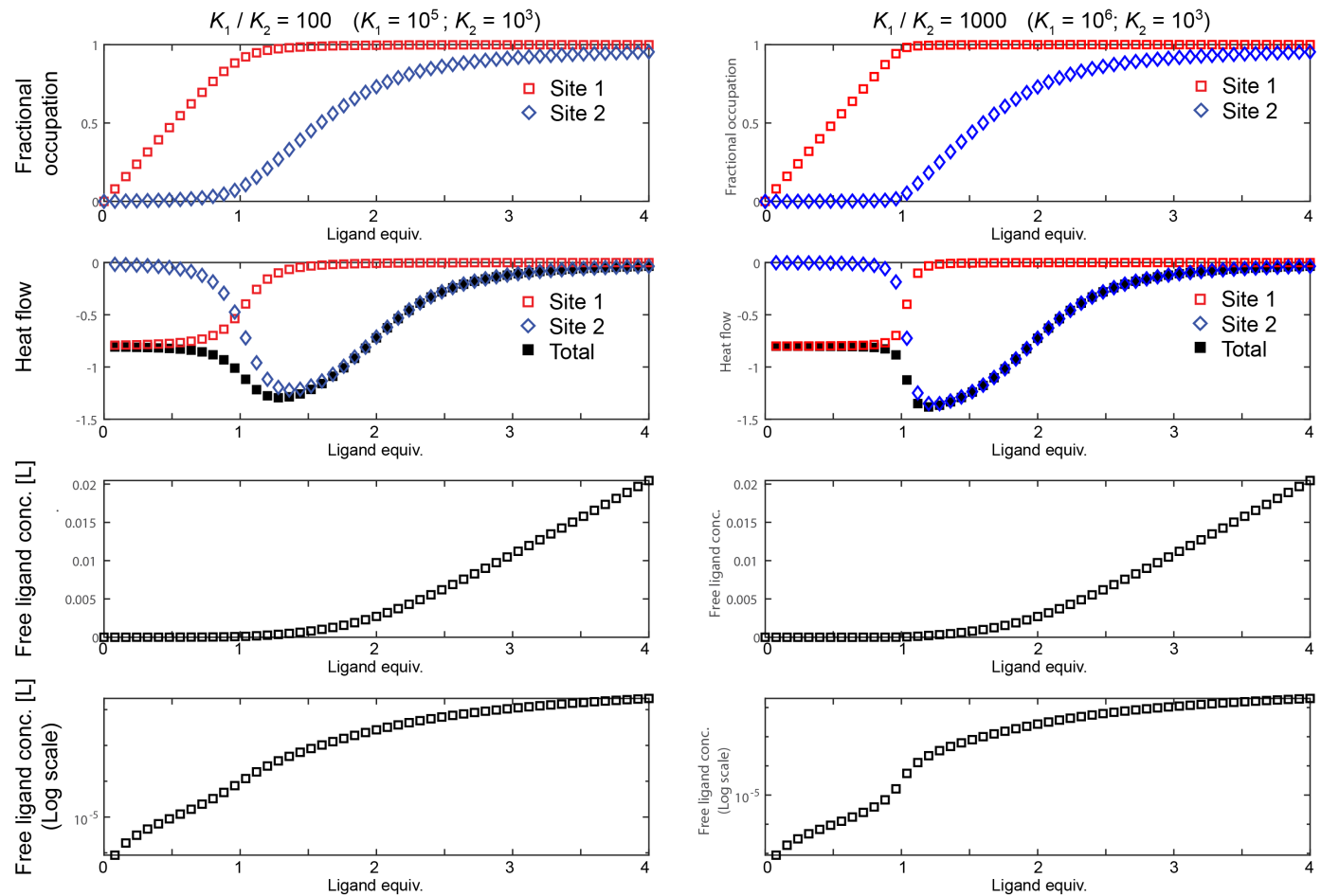

Figure S2: An illustrative model showing binding to two sets of independent sites where sites of type 1 (the stronger binder: $\left.K_{1}>K_{2}\right)$ have a less negative enthalpy of binding than sites of type $2\left(\Delta H_{2}<\Delta H_{1}\right)$. 
In our analysis we found that a minimum of three components, $k$, was necessary to interpret the oleate for alkylphosphonate ligand exchange signals, and this discussion is presented for that case. The backgroundcorrected power signal $P_{n}(t)$ following every injection $n$, with the time $t$ from the start of the injection, can then be written as:

$$
P_{n}(t)=A_{1, n} w_{1}(t)+A_{2, n} w_{2}(t)+A_{3, n} w_{3}(t)
$$

where $A_{k, n}$ is the amplitude of component waveform $w_{k}(t)$ for the $n$th injection. Importantly, the extent of reaction is always positive as the concentration of titrant is increasing, and thus while a given waveform component $w(t)$ might be net endo- or exothermic, the amplitudes of the components cannot change sign: $A_{k, n} \geq 0$. Equation 23 can be expressed more concisely in matrix form:

$$
\mathbf{P}_{n}=\mathbf{W} \mathbf{A}_{n}
$$

where $\mathbf{P}_{n}$ is a vector representing the power at each time $t, \mathbf{W}=\left[\mathbf{w}_{1} \mathbf{w}_{2} \mathbf{w}_{3}\right]$ is a matrix composed of the time response $w_{k}(t)$ for each waveform component, and $\mathbf{A}_{n}$ is a vector of the amplitudes of each titrant addition component $k$ in the response to injection $n$. The total heat associated with each component for each characteristic response is obtained as

$$
q_{k, n}=\int A_{k, n} w_{k}(t) d t
$$

evaluated from the start of injection $n$ to the start of the next.

If the form of the waveform components $w_{k}(t)$ is known, the amplitudes for each injection may then be nominally obtained as the solution to the following matrix equation:

$$
\mathbf{A}_{n}=\mathbf{W}^{-1} \mathbf{P}_{n}
$$

However, as there are many more time points than components considered, this equation is overdetermined and in general a least-squares solution is required. Moreover, the requirement that $A_{k, n} \geq 0$ must be imposed. We achieved this using the Matlab function lsqnonneg, which determines the least-squares optimized solution with non-negative coefficients.

If indeed it was the case that all time responses can be described by a linear combination of $k$ components, then it should have been possible to identify fundamental waveform components $w_{k}(t)$ from the data by considering representative "model" responses or differences between them $m_{k}(t)$ for resultant model waveforms. Data represented in Figure S7 (E-H) demonstrates how model responses were chosen directly from ITC thermograms. Then, for example, we can express proposed $\mathbf{w}_{k}$ as:

$$
w_{k}(t)=m_{k}(t)-r_{k, k^{\prime}} m_{k^{\prime}}(t)-r_{k, k^{\prime \prime}} m_{k^{\prime \prime}}(t)
$$

or

$$
\mathbf{W}=\mathbf{M R}
$$

Where $\mathbf{M}$ contains model waveforms and $\mathbf{R}$ is a $k$ by $k$ matrix. The off-diagonal elements of $\mathbf{R}$ are the parameters by which the set of basis time-response waveform vectors $\mathbf{w}_{k}$ are selected, as demonstrated in Figure S8. The amplitude $\mathbf{A}_{k, n}$ for each component in each response is computed according to Equation 26, and used to obtain a fit $\mathbf{P}_{n}=\mathbf{W} \mathbf{A}_{n}$ for each response. The optimum basis waveform set $\mathbf{w}_{k}$ minimizes the error between the reconstructed thermogram power signal $\mathbf{P}_{n}$ and the ITC thermogram data over the course of the entire titration. The separated heat responses $q_{k, n}$ are obtained for each component $k$ from Equation 25, along with any unfit heat indicated by the difference between $\sum_{k} q_{k, n}$ and the integral of the total integrated power signal for each injection $n$. 


\section{Fitting of heat signals to obtain model parameters}

For each ITC trial titration, best-fit parameters for thermodynamic models ( $K, \Delta H$, and $N$ for Langmuir; or $K_{1 \text {,eff }}, \Delta H_{1}, N_{1}, K_{2}, \Delta H_{2}$, and $N_{2}$ for two sets of independent sites) were identified based on the heat signals associated with each injection. In particular, the parameters were varied so as to minimize the sum of the squares of the differences between model predictions and measured heats. In cases where the total heat signals were separated into components as described above, the squared errors for each component could be added together to optimize with respect to all components simultaneously. Minimization of the total error with respect to model parameters was conducted using the fminsearch function in Matlab. For the initial Langmuir model this was accomplished directly using the three free parameters $(K, \Delta H$, and $N$ ). For the two types of independent sites, three-parameter fits to each set of site parameters (Site 1 and Site 2) were accomplished separately in an iterative fashion until there was no significant further change in parameter values. As is customary in ITC analysis, the first two injection signals (which are often perturbed by premixing of reagents) were excluded from the analyses.

Within this general approach, as discussed in the main text, interpretation of the data in terms of the two independent sets (types) of sites model was accomplished by considering the total heat associated with rapid basis waveforms $\mathbf{w}_{1}$ and $\mathbf{w}_{2}$ together as depending on the change in occupancy at Site 1, and heat associated with the basis waveform for the slow exotherm $\mathbf{w}_{3}$ as depending on the change in occupancy at Site 2:

$$
\begin{aligned}
& V \Delta\left[\mathrm{L}_{2}\right]_{\mathrm{b} 1} \Delta H_{1}=q_{1, n}+q_{2, n} \\
& V \Delta\left[\mathrm{L}_{2}\right]_{\mathrm{b} 2} \Delta H_{2}=q_{3, n}
\end{aligned}
$$

Where $V$ is the volume of the ITC reaction cell, and $\left[\mathrm{L}_{2}\right]_{b 1}$ and $\left[\mathrm{L}_{2}\right]_{b 2}$ represent the concentrations of incoming (alkylphosphonic acid) ligand bound at Sites 1 and 2 respectively.

\section{1 "Characteristic" analysis: using slow exotherm $\left(q_{3, n}\right)$ to constrain two types of indepen- dent sites model}

The full set of titration injections was considered, but only the differences between measured $q_{3, n}$ (the heat associated with the slow exothermic waveform $\mathbf{w}_{3}$ ) and the model for Site 2 are used to guide the fit. In particular, $q_{3}$ is used to find optimized values for $K_{1 \text {,eff }}, N_{1}, K_{2}, \Delta H_{2}$, and $N_{2}$. The Site 2 signal does not depend on $\Delta H_{1}$ : therefore, after optimizing the other parameters, $\Delta H_{1}$ is solved in a single-parameter fit to best match the measured Site 1 heat. Ultimately the fit is "pinned," analogous to an overall average enthalpy of exchange.

\section{2 "Limiting" analysis: using both Site 1 and Site 2 heats to constrain two independent types of sites model near equivalency point}

A limited set of injections, not beginning until $\left[\mathrm{L}_{2}\right]_{t} \approx N_{1}[\mathrm{M}]_{t}$, is considered. Within this set of sampled points, parameters for the two independent types of sites model are iteratively solved by comparison of the predicted heat signals for $q_{1, n}+q_{2, n}$ (Site 1) and $q_{3, n}$ (Site 2) to the titration experiment results. 


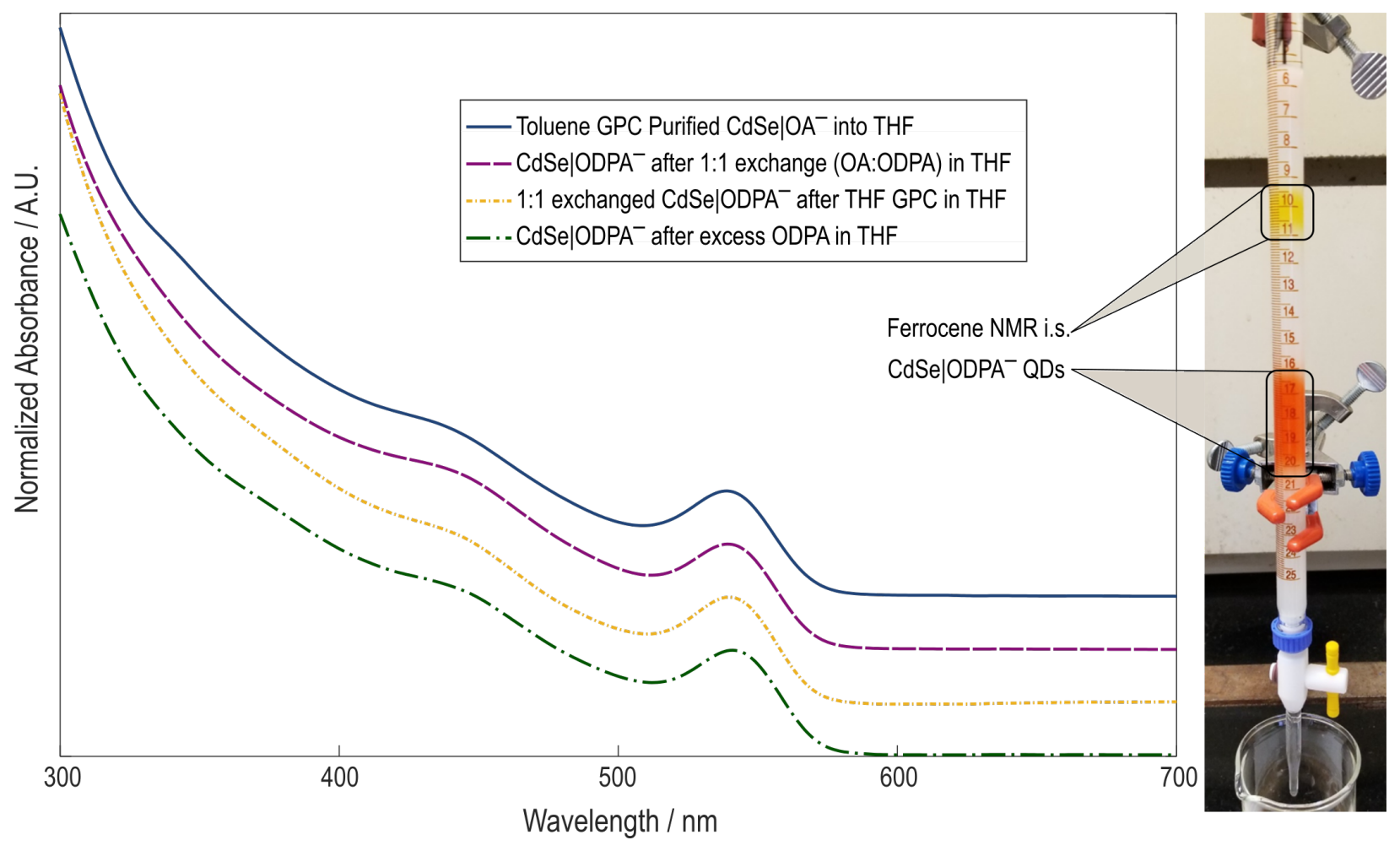

Figure S3: UV/Vis absorption spectra of CdSe QD solutions before and following oleate-to-phosphonate ligand exchange as well as subsequent re-purification. The absorption profiles, normalized at the lowest energy excitonic peaks and vertically offset for clarity, show consistent lowest-energy absorpption peak shapes and positions indicating that no degradation of the QDs occurred during the ligand exchange titration nor upon re-purification. Photo shows anhydrous THF GPC re-purification step in progress: the yellow band is the ferrocene ${ }^{1} \mathrm{H}$ NMR internal standard, which is clearly resolved (indicative of progression of small molecules) from the orange band of phosphonate ligand-exchanged QDs. 

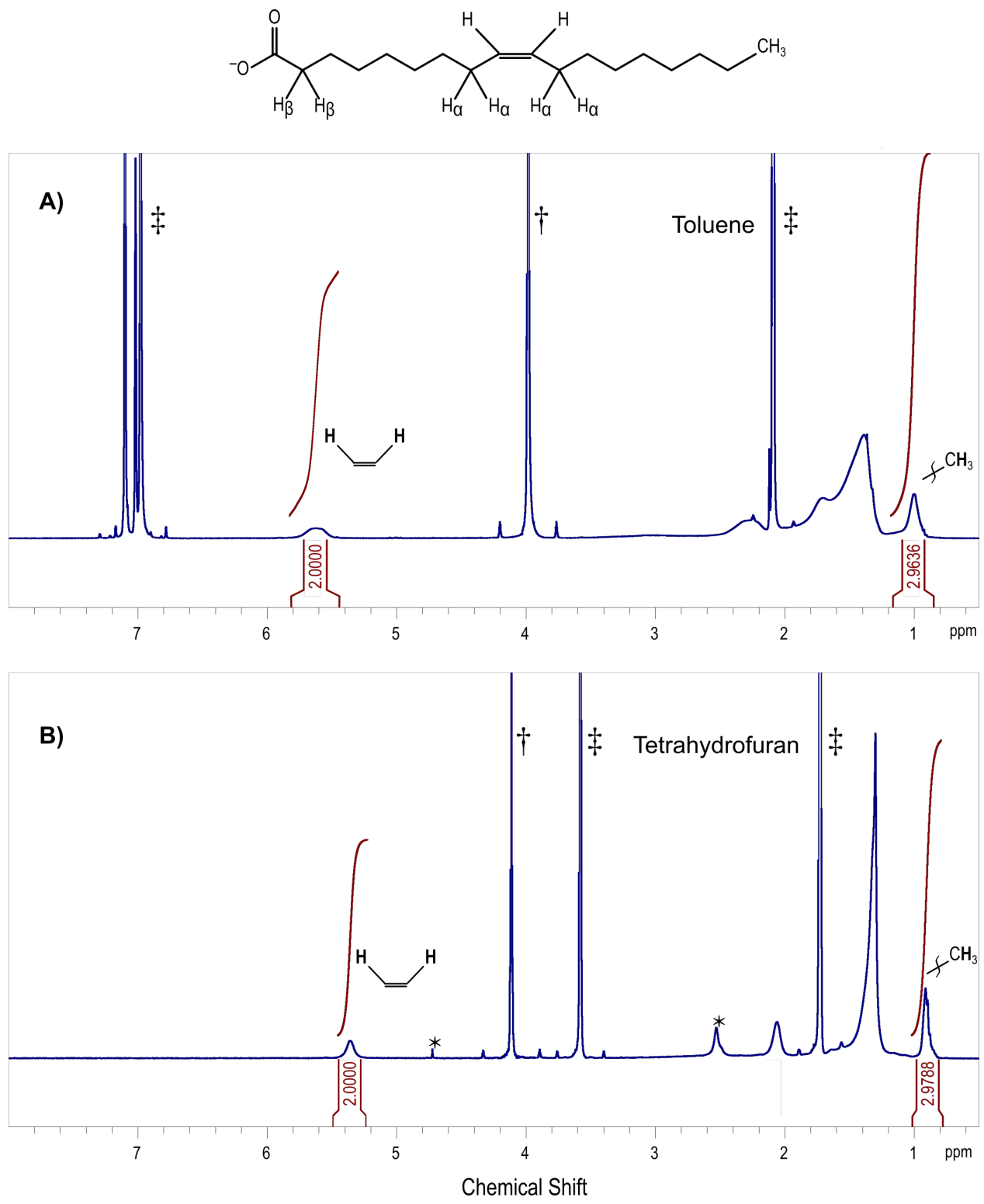

Figure S4: Full ${ }^{1} \mathrm{H}$ NMR spectra of oleate-capped CdSe QDs purified by GPC with (A) Toluene m.p. and subsequently brought into [D8]Toluene, or (B) anhydrous THF m.p. into [D8]THF. †Ferrocene internal standard. $\ddagger N M R$ solvent peaks. ${ }^{*} \mathrm{H}_{2} \mathrm{O}$ impurity peaks. In both spectra, integration shows a 2:3 ratio of the vinyl-to-methyl proton peaks, as expected if oleate is the sole ligand species bound to the QD surface. 

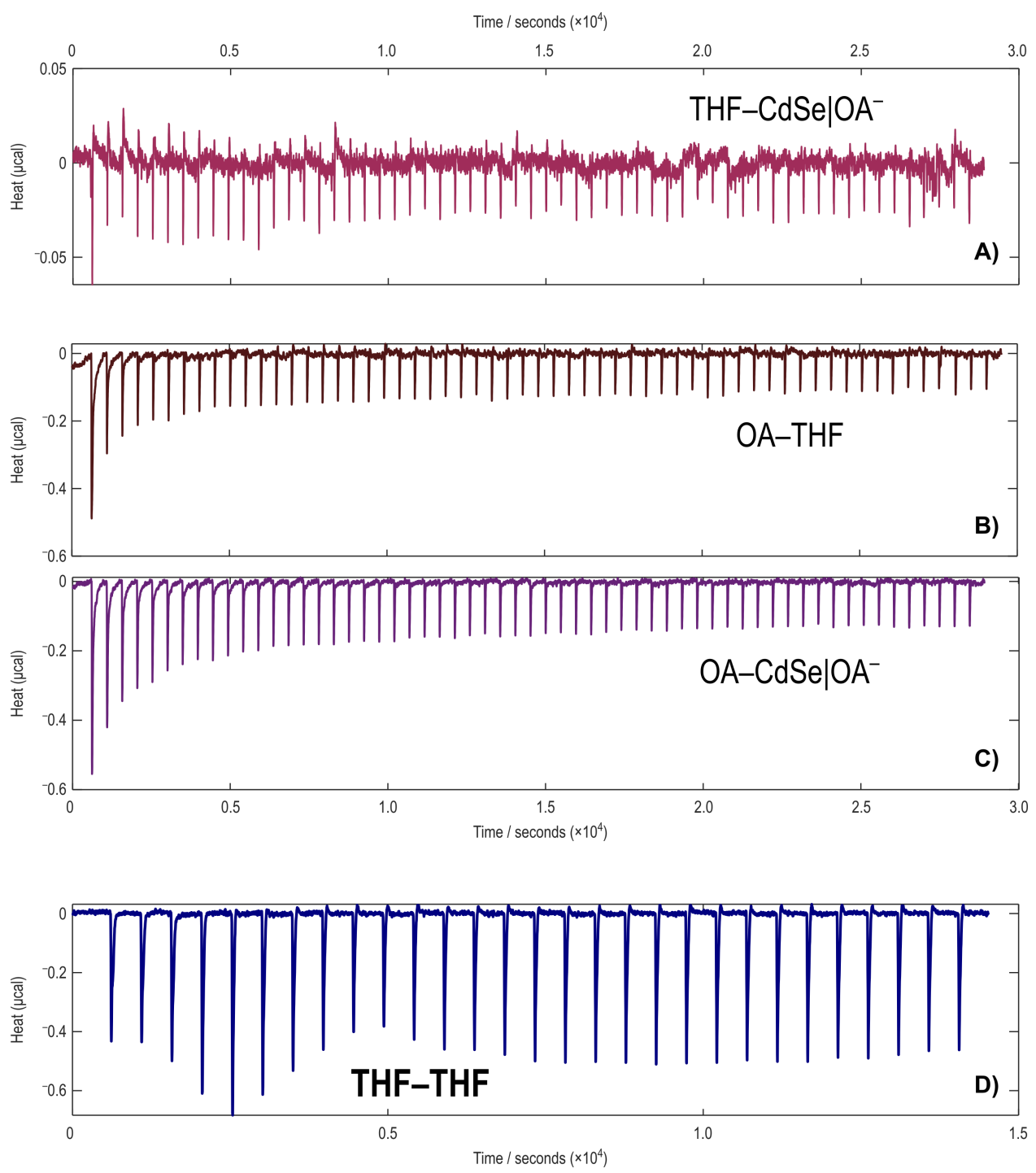

Figure S5: Several reference titrations were performed for comparison to and deconvolution from ligand exchange results as discussed in narrative, as all titration components contribute indriscriminantly to the evovled heat within the ITC system. (A) anhydrous THF solvent-to-CdSe $\mid \mathrm{OA}^{-}$QDs (note narrower heat scale, y-axis); (B) OA ligand-to-anhydrous THF solvent; (C) OA ligand-to-CdSe $\mid \mathrm{OA}^{-}$QDs titration illustrating entirely exothermic signal of comparable magnitude to that of the OA-THF control in (B), which could also advise on expected heat signals for extruded OA from the ligand-exchange QDs; and (D) anhydrous THF solvent-to-solvent titration (note expanded timescale) Each of these reference titrations were deemed negligible, as compared to the anhydrous THF-THF control (D), which established the ITC mechanical heat baseline. 

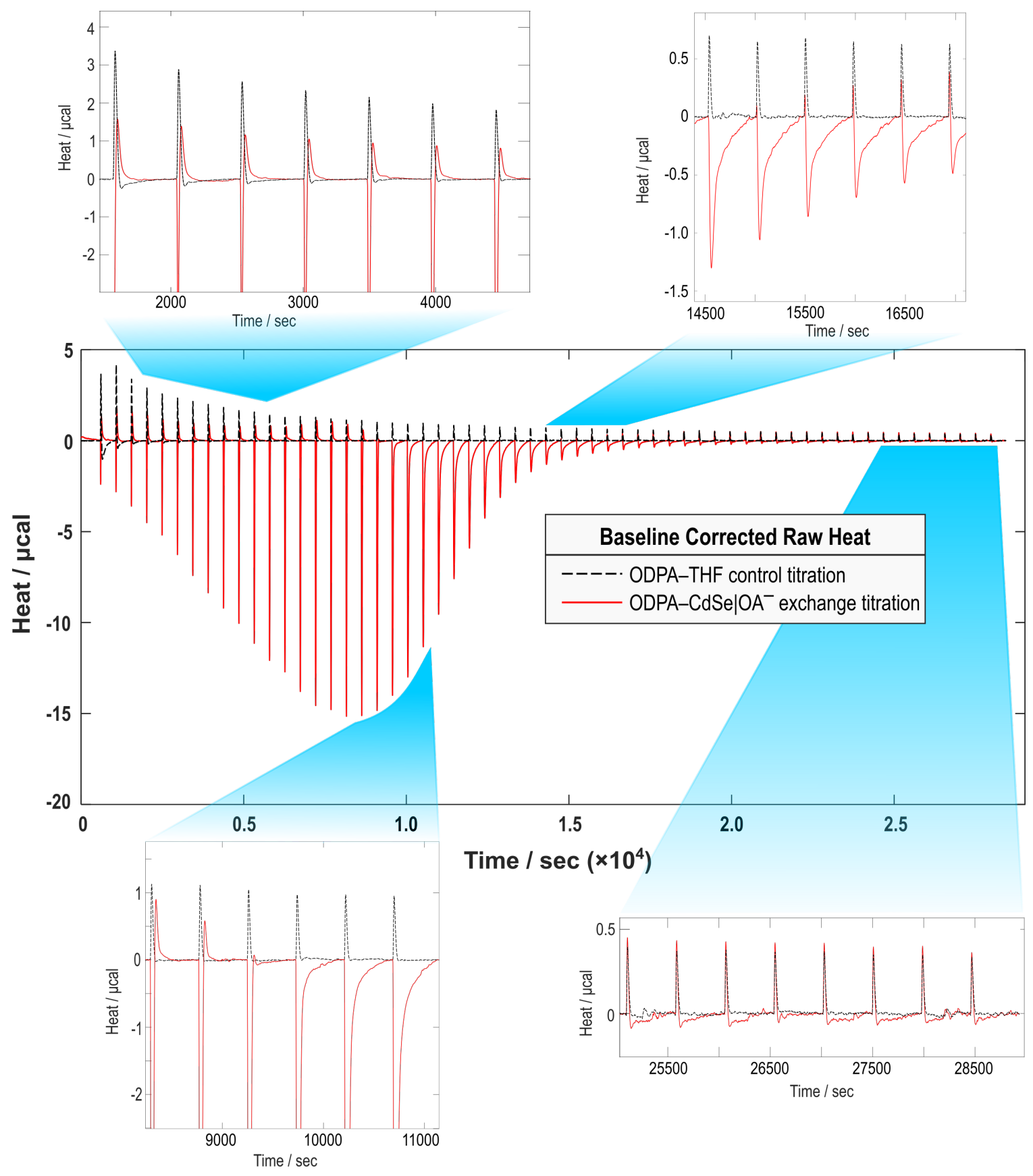

Figure S6: Representative baseline-corrected ODPA-CdSe $\mid \mathrm{OA}^{-}$ligand exchange thermogram (red solid line) overlaid with control ODPA-to-anhydrous THF solvent thermogram (black dashed line). The ODPATHF titration describes a significant heat of dilution underlying the exchange reaction of interest. Vignettes offer magnified views of regimes featuring initial rapid exo- and endo-thermic signals (assigned as anionic exchange, Site 1), which transition to slow exothermic signals (assigned as ODPA association, Site 2). The slow exotherm diminishes as ODPA association sites become saturated, and ultimately the reaction thermogram peaks reveal a return to correspondence with control titration thermogram peaks. The control ODPATHF dilution-correction titration was subtracted from exchange titrations for analysis. Consistent heat onset observed in the ODPA-THF titration injections also provided the ITC instrument response function (IRF) for reaction kinetics insights and integrated isotherm modeling. 

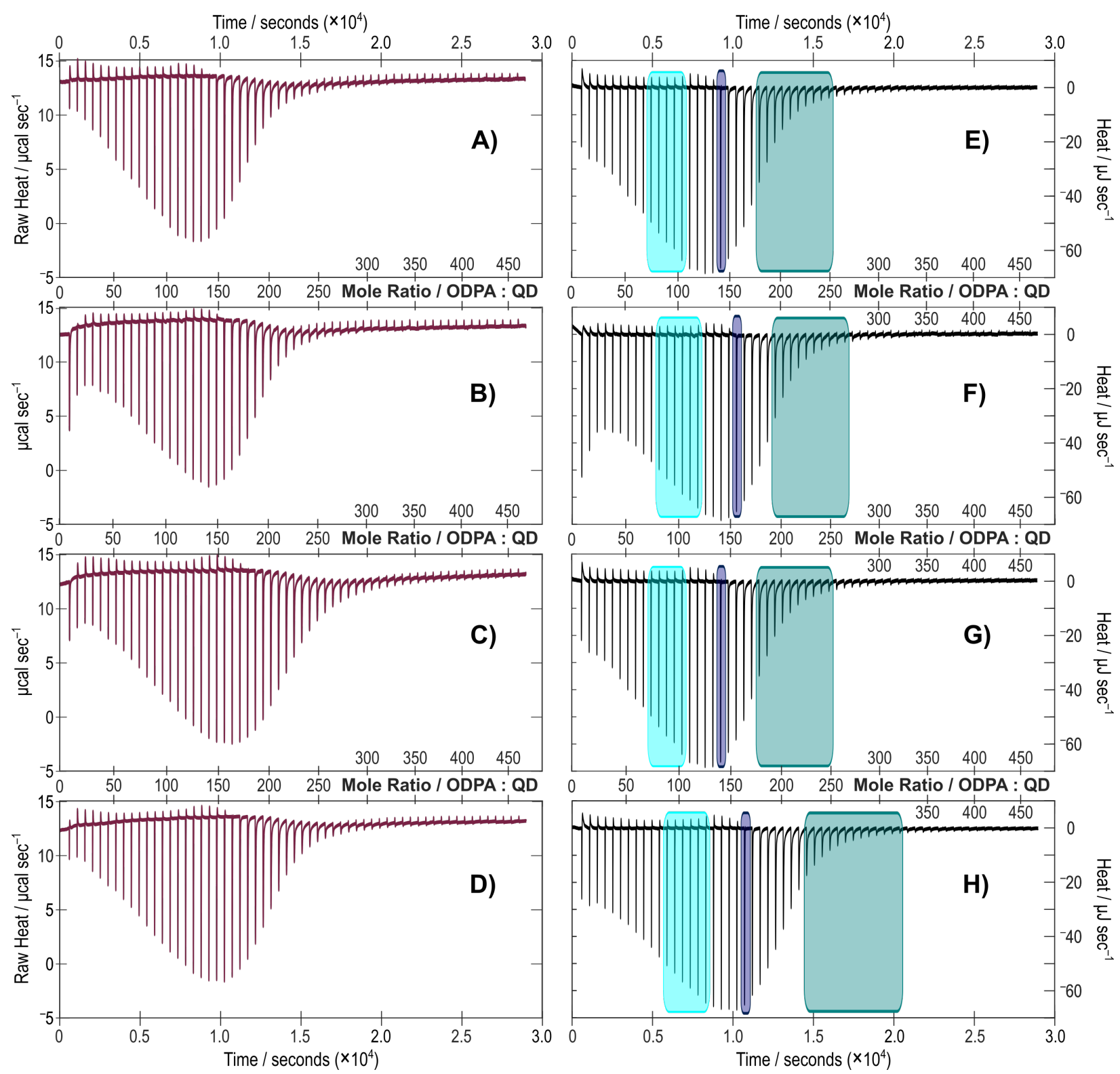

Figure S7: (A-D) Replicate titrations of ODPA into CdSe $\mid \mathrm{OA}^{-}$following GPC purification in Toluene m.p., comparable to Figure 1 in main text. These thermograms show raw heat data before baseline correction and reference titration subtraction (dilution-correction). (E-H) Thermograms to the right are baseline- and dilution- corrected transforms of the corresponding raw heat thermograms (A-D). The shadings in thermograms (E-H) indicate the exact injections used to construct that trial's waveform models and consequent basis waveforms: (left to right) aqua populates waveform component $2\left(w_{2}\right)$, navy populates waveform component $1\left(w_{1}\right)$, and teal populates waveform component $3\left(w_{3}\right)$; as further demonstrated in Figure S8. 

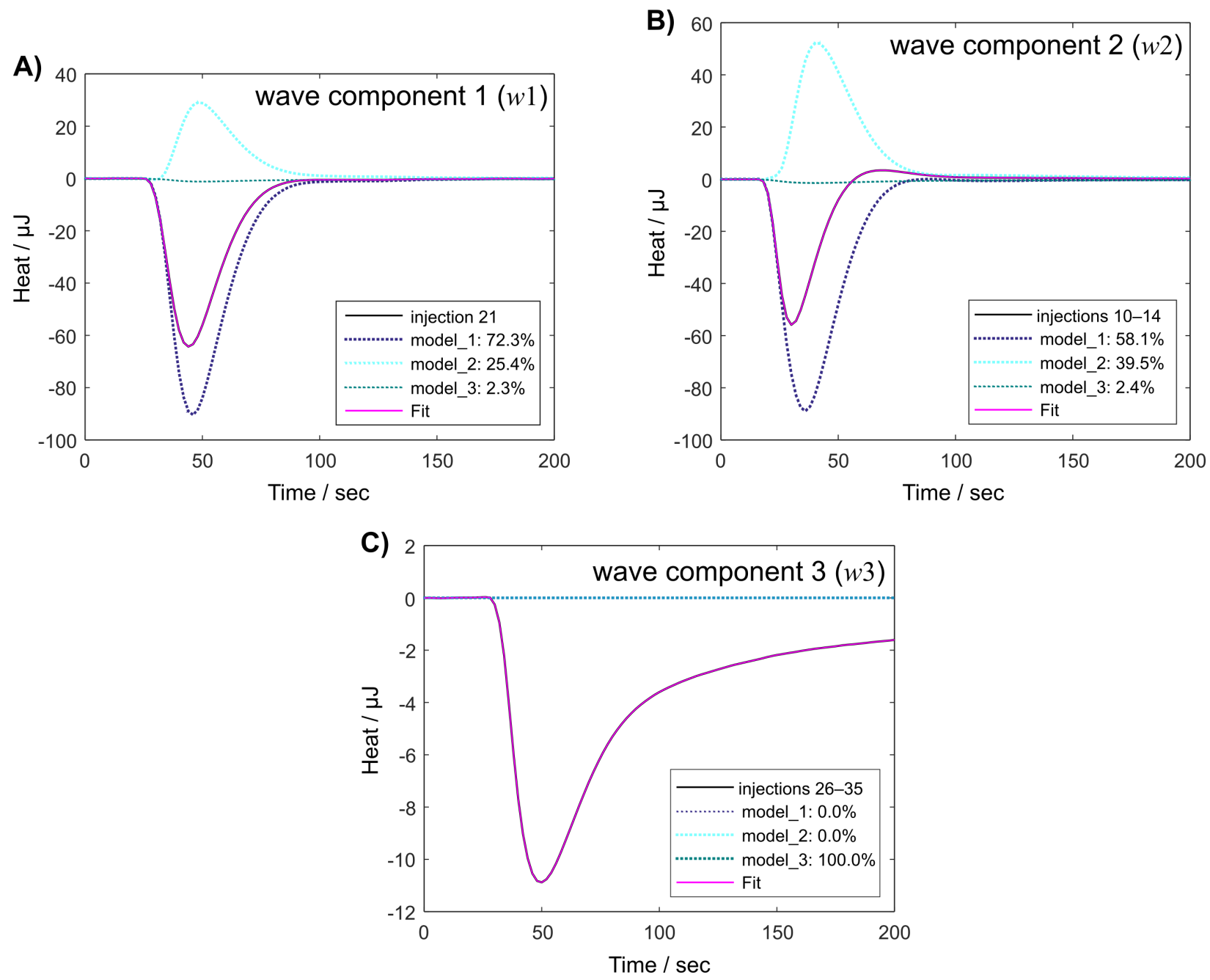

Figure S8: Representative identification of 3 basis waveforms (A) $w_{1}$, (B) $w_{2}$ and (C) $w_{3}$ from different representative thermogram injection responses in the exchange reaction titration shown in Figure 1 and detailed in Figure S7 (E-H). Here injection responses used as models for $w_{1}$ and $w_{2}$ are considered to each contain contributions from both $w_{1}$ and $w_{2}$ waveform models, but in different proportions shown as relative percentages in the legends on each waveform plot; ultimately resulting in the linearly independent basis waveforms. Contrarily, $w_{3}$ is obtained directly and solely from later thermogram injection responses. 


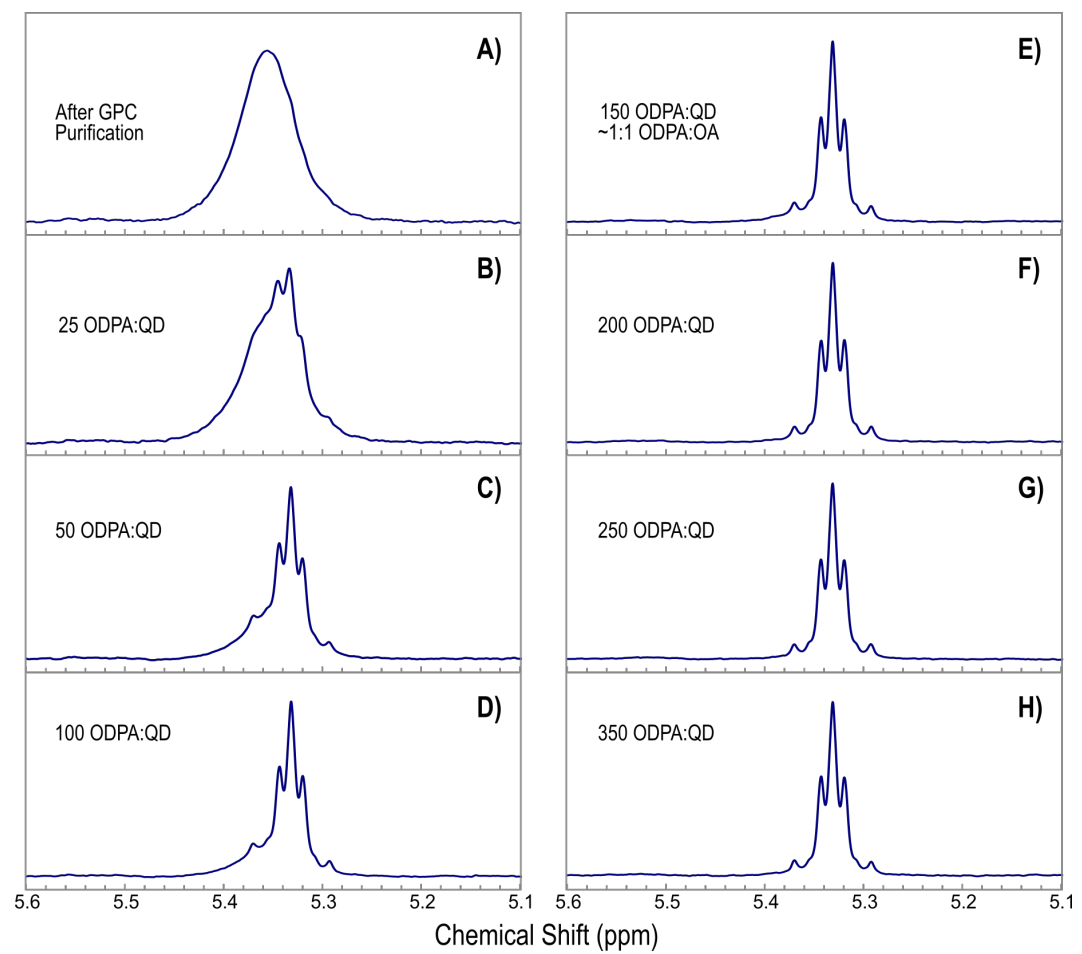

Figure S9: Vinyl region of ${ }^{1} \mathrm{H}$ NMR spectra for CdSe $\mid \mathrm{OA}^{-}$QDs after Toluene GPC and subsequently brought into [D8]THF. Equivalents of ODPA were introduced analogous to the calorimetric titrations in Figures $1 \& 3$ and coinciding with the ${ }^{31} \mathrm{P}\left\{{ }^{1} \mathrm{H}\right\}$ NMR titration spectra $\left({ }^{1} \mathrm{H}\right.$ NMR insets) in Figure 2 of the narrative. Panel (E) at $~ 1: 1$ ODPA-to-OA equiv. upon maginification reveals the persistent downfield and deshielded broadened shoulder indicative of remaining $\mathrm{OA}^{-}$bound to the QD surface.

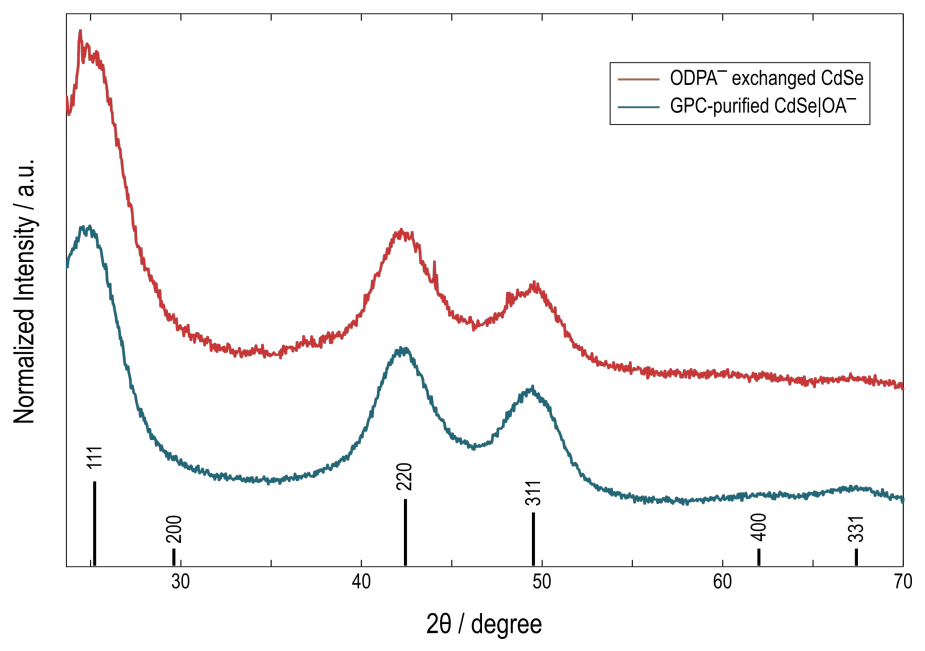

Figure S10: PXRD of CdSe $\mid \mathrm{OA}^{-}$QDs before (bottom) and after (top, $\mathrm{CdSe} \mid \mathrm{ODPA}^{-}$) ligand exchange with ODPA. Database Bragg reflections for zincblende CdSe are shown for comparison; and confirm that the complete quantitative ligand exchange does not induce an internal crystal transformation. 

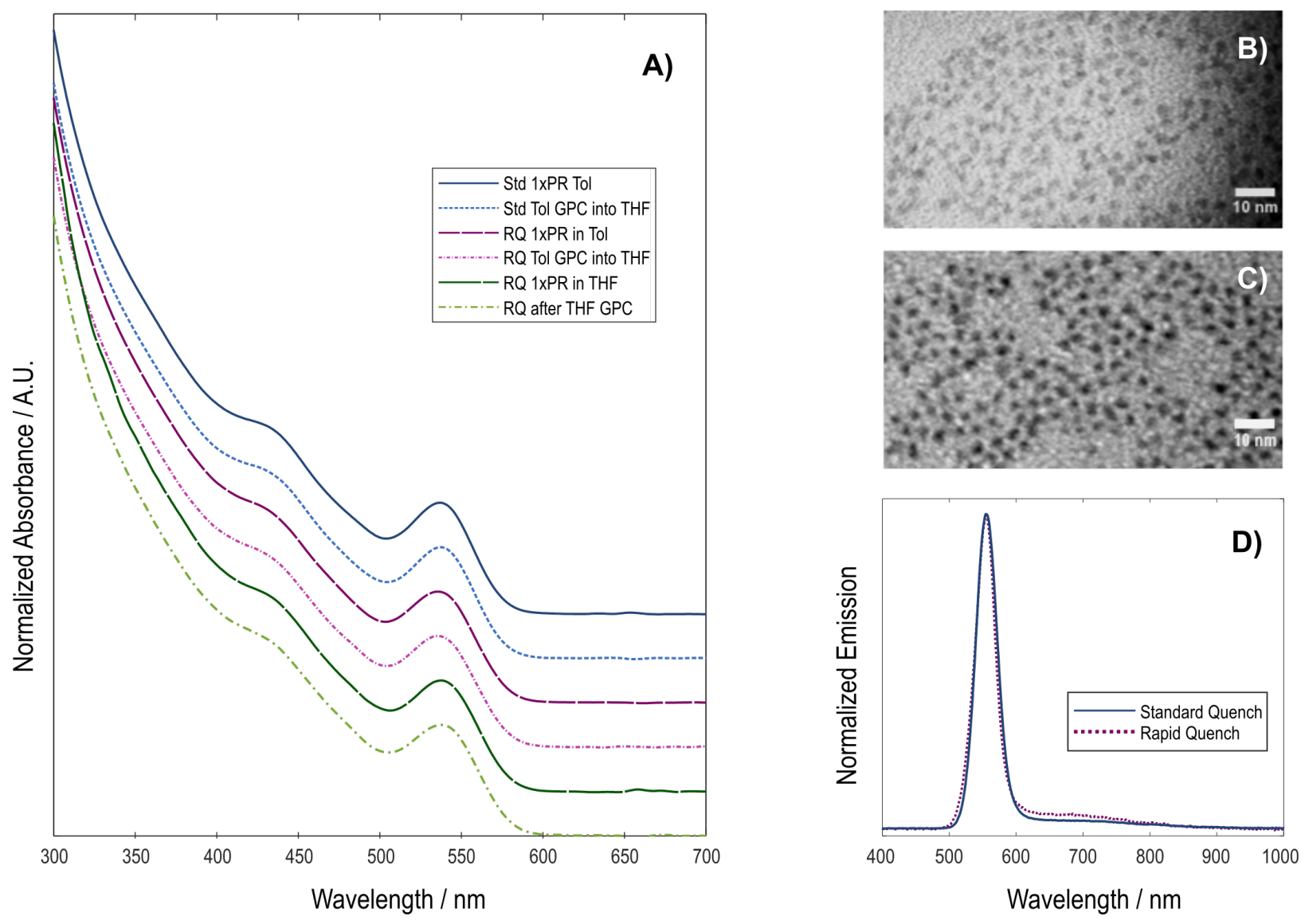

Figure S11: (A) UV/Vis absorption for Standard and Rapid Quench CdSe $\mid \mathrm{OA}^{-}$samples prior to, and ensuing from, either toluene or THF mobile phase GPC purification. Spectra are normalized according to the lowest-energy absorption peak and vertically offset for clarity. (B,C) TEM images of the (B) Standard and (C) Rapid Quench QDs. (D) Steady state PL emission also for both synthetic preparations. Both absorption and emission spectra are normalized to their lowest energy extinction peaks. The typically employed optical measurements for QD characterization show no discernible difference between samples regardless of synthetic prepration, despite drastic surface chemistry differences detected by ITC. 


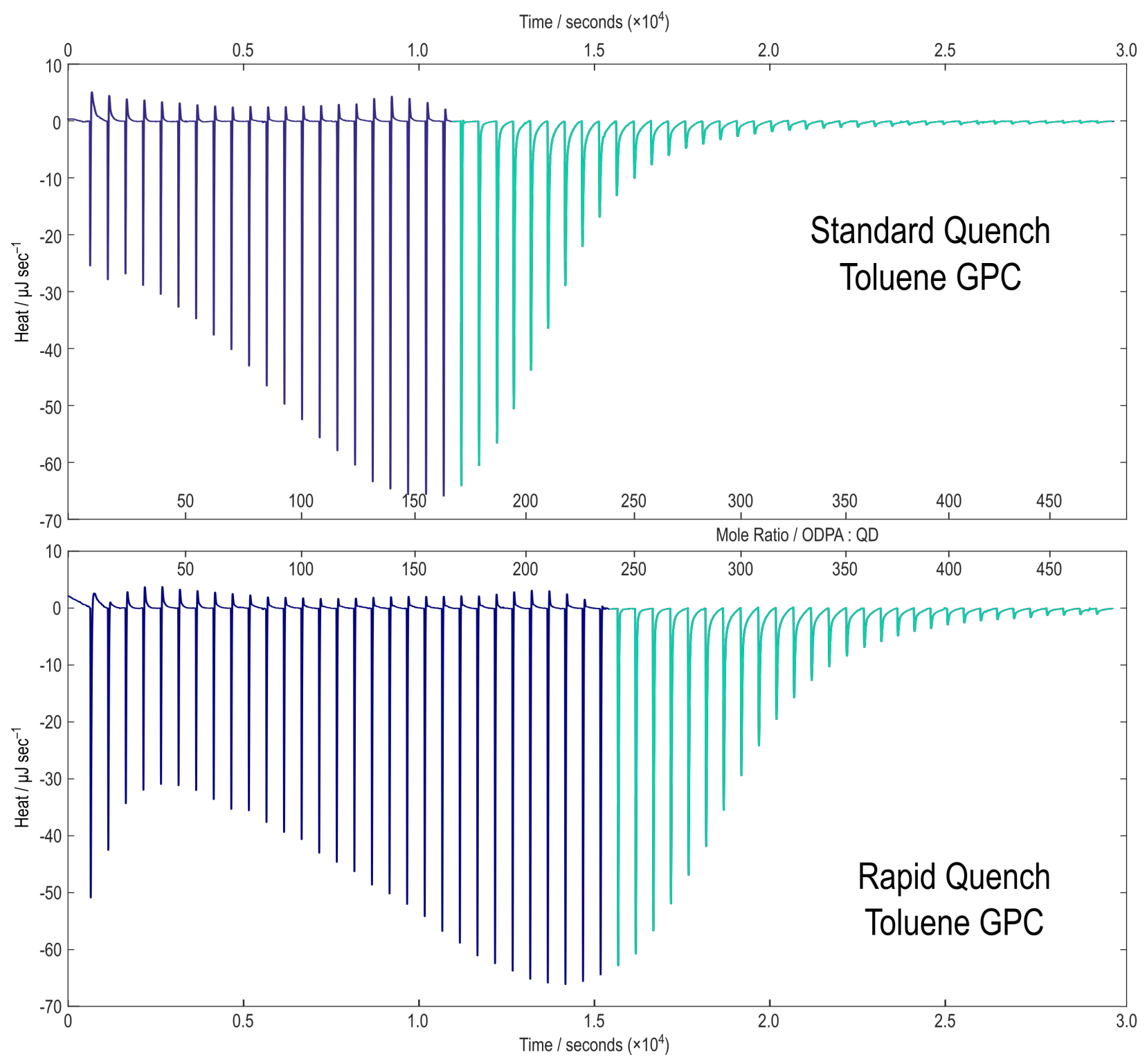

Figure S12: Representative thermograms for ODPA exchange of oleate-capped CdSe QDs formed by slower (top, Std Quench) or rapid (bottom, RQ) thermal quenching of the synthetic reaction. In the thermograms initial navy-colored injection peaks indicate ODPA-for-OA exchange (Site 1) and ensuing teal-colored inections peaks indicate ODPA association interaction (Site 2) analogous to the thermograms in Figure 4 of the narrative. According to typical characterization methods as demonstrated in Figure S11, the CdSe $\mid \mathrm{OA}^{-}$ QD starting materials are of apparently indistinguishable size. However, the apparent endpoints for Site 1 detected with ITC suggest at least a different surface chemistry imparted by the different synthetic thermal quenching. 


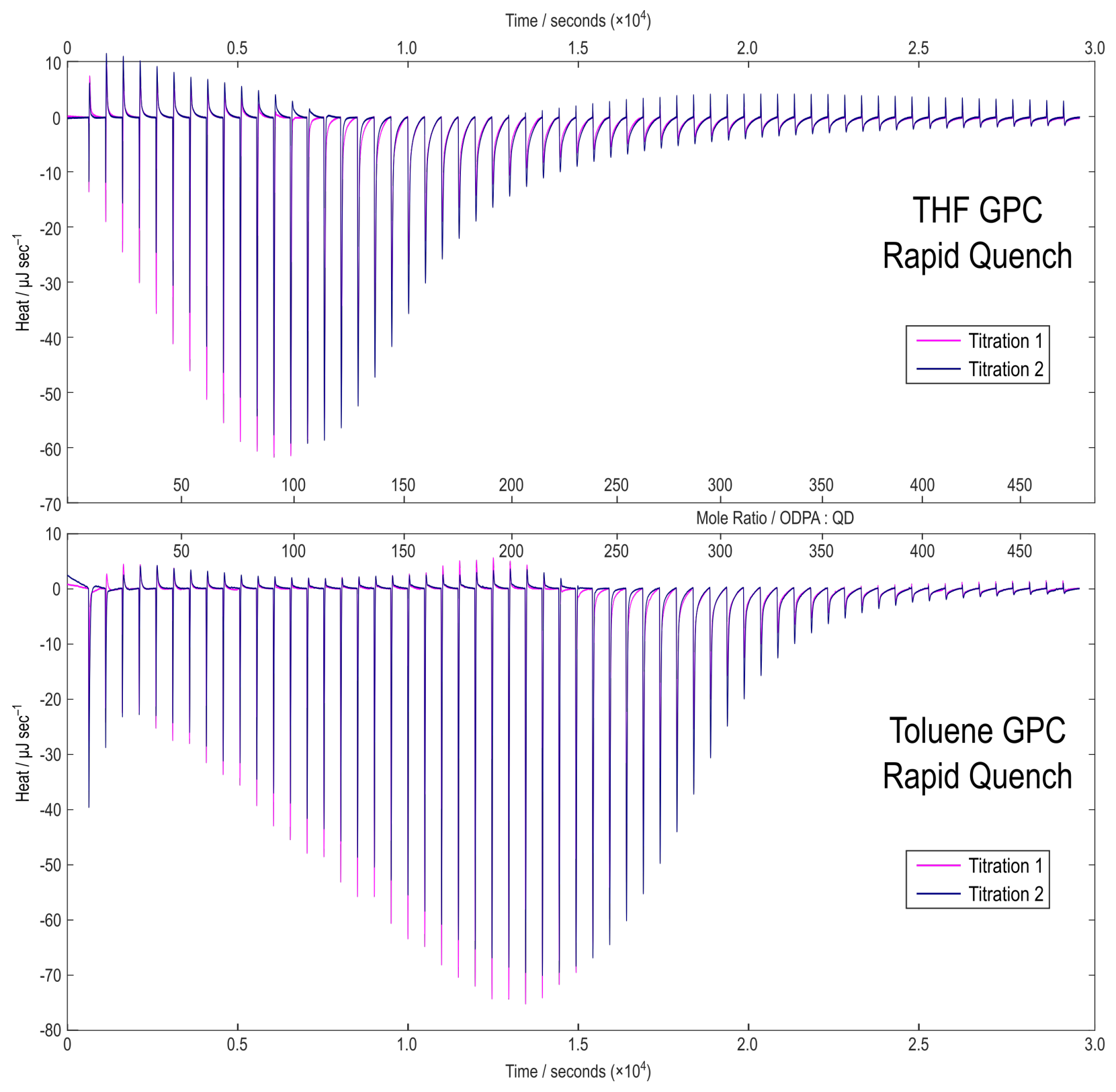

Figure S13: Replicate overlaid thermograms for ODPA-CdSe $\mid \mathrm{OA}^{-}$following GPC purification with anhydrous THF (top) or Toluene (bottom) mobile phase, comparable to Figure 4 in the main text. All thermogram curves are independent experimental datasets for the Rapid thermal Quench (RQ) synthetic procedure. 


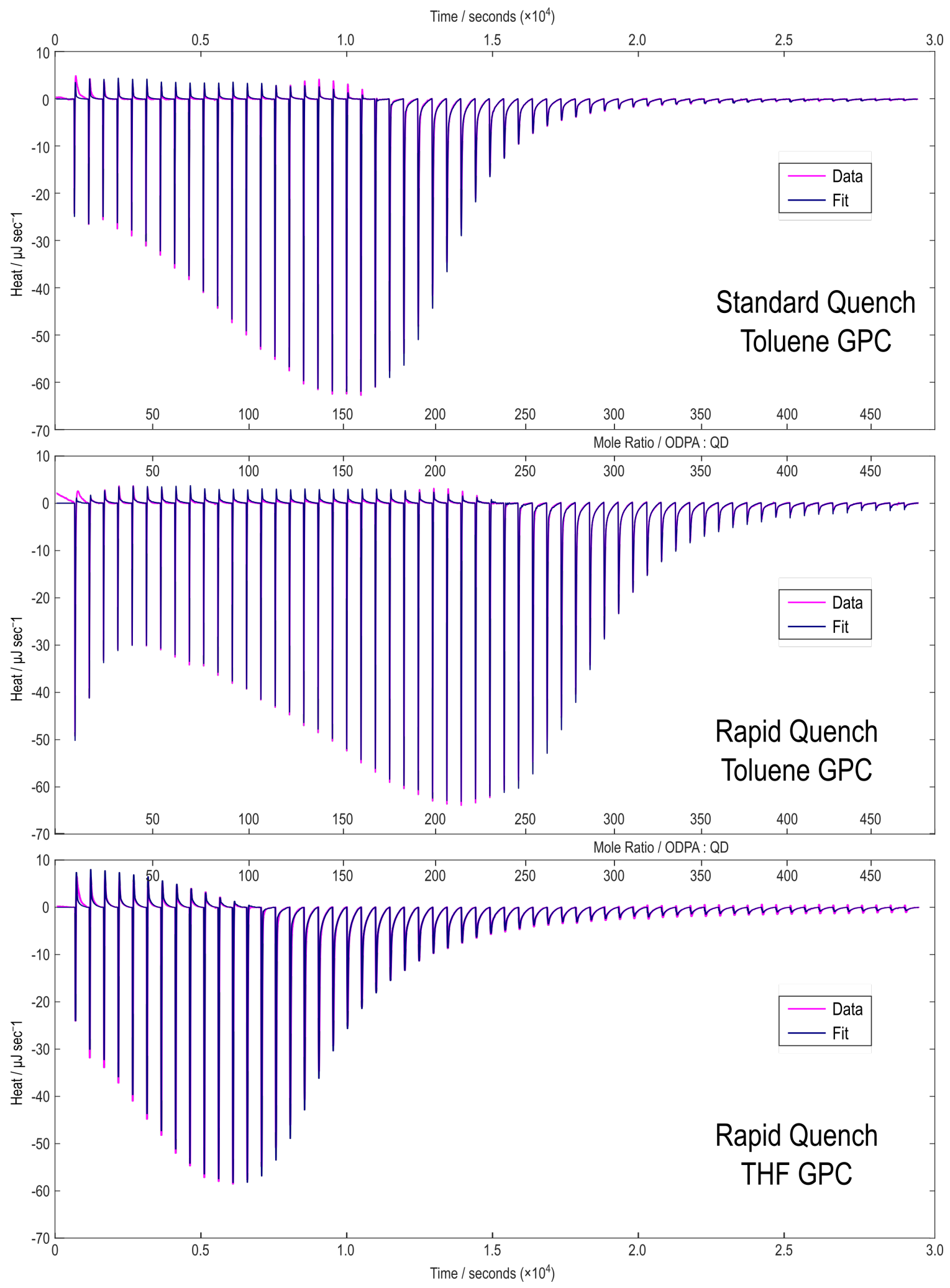

Figure S14: Representative thermograms for each of the sample preparation variations, showing complete waveform fits from time trace analysis as described in Section 2 overlaying the original data. 

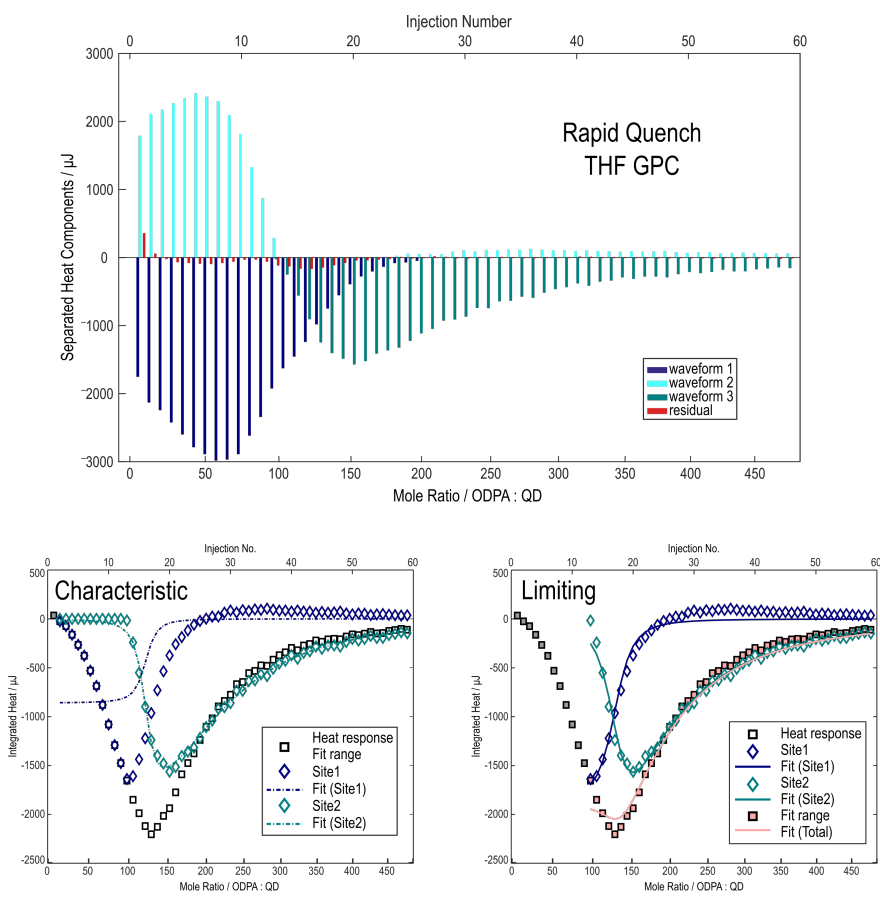

Figure S15: Themodynamic model results for ODPA-CdSe $\mid \mathrm{OA}^{-}$titration of RQ QDs following anhydrous THF GPC, comparable to Figure 3 and corresponding to the thermogram in Figure 4A in the main text.
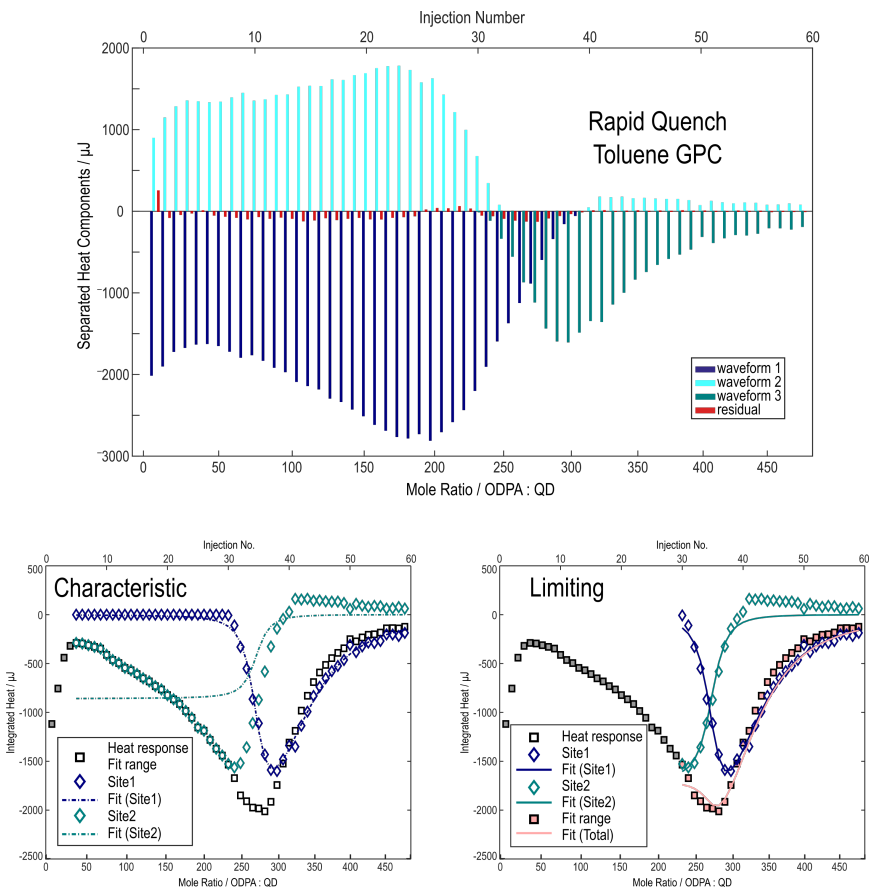

Figure S16: Themodynamic model results for ODPA-CdSe $\mid \mathrm{OA}^{-}$titration of RQ QDs following Toluene GPC, comparable to Figure 3 and corresponding to the thermogram in Figure 4B in the main text. 
Table S1: Outlier test for titration of $\mathrm{CdSe} \mid \mathrm{OA}^{-}$with ODPA at 2 2x QD concentration

\begin{tabular}{lcccc}
\hline Parameter & $K_{\text {exch }}$ & $N_{1}$ & $\Delta H_{1}$ (Char.) & $\Delta H_{1}$ (Lim.) \\
\hline Concentrated $\left[\mathrm{CdSe} \mid \mathrm{OA}^{-}\right]$ & $47.1 \times 10^{2}$ & 196 & $-5.5 \mathrm{~kJ} \mathrm{~mol}^{-1}$ & $-13.0 \mathrm{~kJ} \mathrm{~mol}^{-1}$ \\
\hline Q value & \\
\hline${ }^{a}$ Statistical test for outliers as compared to $Q_{\text {crit }}$ & 0.327 & 0.821 for $99 \%$ confidence level on 5 observations.
\end{tabular}

Table S2: Brandt's $c$ parameter for Site 2 thermodynamic parameters

\begin{tabular}{lcccc}
\hline Sample ID & Std. Tol. GPC & Std. 2 x initial [QD] & RQ Tol. GPC & RQ THF GPC \\
\hline$c$ parameter & 3.5 & 2.5 & 3.3 & 2.7 \\
\hline
\end{tabular}

